\title{
Rotational relaxation times from Raman band profiles for benzonitrile in different solvents
}

\author{
H A AHMED and G THYAGARAJAN* \\ Department of Physics, Indian Institute of Technology, Powai, Bombay 400076, India
}

\begin{abstract}
The Raman band profiles of two vibrational bands of benzonitrile belonging to the $a_{1}$ species ( 2243 and $1000 \mathrm{~cm}^{-1}$ ) were analysed by separating the isotropic and anisotropic components. Spectra of benzonitrile were recorded, using argon laser excitation, in solutions of $\mathrm{CCl}_{4}, \mathrm{CS}_{2}$, cyclohexane, cyclohexanol and tertiary butyl alcohol -- all having different viscosities. The rotational correlation times were evaluated from the band profiles. The results indicate that (a) the isotropic (and not the anisotropic) linewidth increases with increasing viscosity, which is attributed to the greater effect of intermolecular interactions on vibrational relixation; and (b) the two modes of the same species give different relaxation times.
\end{abstract}

Keywords. Rotational relaxation times; benzonitrile; Raman band profiles; vibrational bands; isotropic and anisotropic components.

\section{Introduction}

Among the techniques available for the study of molecular motions in liquids, the Raman scattering technique has the distinct advantage that it enables simultaneous analysis of both reorientational and non-reorientational processes (Naffie and Peticolas 1972). Using the depolarized spectrum of totally symmetric vibrational bands, a separation of vibrational (isotropic part) and rotational (anisotropic part) contributions to the width of the band can be made. Further, in Raman scattering, the rotational correlation function is essentially a single particle correlation function as the scattering is incoherent and pair correlation contribution to the Raman scattering intensity does not figure in it owing to the random phases of vibrational wavefunctions of different particles.

The correlation functions, $C_{\mathrm{v}}(t)$ (vibrational) $C_{\mathrm{rot}}(t)$ (rotational) and $C_{\mathrm{s}}(t)$ (single particle) are given by

$$
\begin{aligned}
& C_{\text {iso }}(t)=C_{\mathrm{v}}(t)=f_{\text {band }} I_{\text {iso }}(\omega) \exp (i \omega t) \mathrm{d} \omega, \\
& C_{\text {aniso }}(t)=C_{\text {rot }}(t) \times C_{\text {iso }}(t)=f_{\text {bano }} I_{\text {aniso }}(\omega) \exp (i \omega t) \mathrm{d} \omega, \\
& C_{\text {rot }}(t)=\frac{C_{\text {aniso }}(t)}{C_{\text {iso }}(t)}, \\
& C_{s}(t)=C_{\text {rot }}(t) \exp \left[-t\left(\frac{1}{\tau_{\text {rot }}}-\frac{1}{\tau_{\text {iso }}}\right)\right]
\end{aligned}
$$

\footnotetext{
* For correspondence
} 
and

$$
\tau_{\text {aniso }}^{-1}=\tau_{\text {iso }}^{-1}+\tau_{\text {rot }}^{-1}
$$

where $I_{\text {iso }}(\omega)=I_{\|}(\omega)-\frac{4}{3} I_{\perp}(\omega)$ and $I_{\text {aniso }}(\omega)=I_{\perp}(\omega)$, in which $I_{\|}$and $I_{\perp}$ stand respectively for the parallel and perpendicular components of polarization of a Raman band. The above relations are based on the following assumptions (Naffie and Peticolas 1972):

(i) The vibrational and rotational correlation functions are statistically independent, as given by the product form of these in (2).

(ii) The correlation functions of both the isotropic and anisotropic parts have the same form of time dependence, namely, exponential decay functions, which implies that

$$
e^{-t / \tau_{\text {aniso }}}=e^{-t / \tau_{\text {iso }}} e^{-t / \tau_{\text {rot }}}
$$

(iii) The band shapes are Lorentzian which implies exponential correlation function in (ii) above and which implies

$$
\left(\Delta \omega_{1 / 2}\right)_{\mathrm{aniso}}=\left(\Delta \omega_{1 / 2}\right)_{\mathrm{iso}}+\left(\Delta \omega_{1 / 2}\right)_{\mathrm{rot}}
$$

(iv) The rotational diffusion constant for rotation of the symmetry axis is given by $D_{\perp}=1 / 6 \tau$.

The present work is based on the application of the above considerations to the Raman bands of benzonitrile taken in different solvents.

\section{Experiment}

The Raman spectra were recorded using a Jobin-Yvon Ramanor HG.2S, spectrophotometer. The exciting source used was an argon-ion laser, Coherent Innova model 70 , with $4 \mathrm{~W}$ maximum power operating at $4800 \AA$ and $5145 \AA$. The spectral slit width was less than $2 \mathrm{~cm}^{-1}$, and the slit effect was corrected according to the equation (Tanabe and Hiraishi 1980a)

$$
\delta_{t}=\delta_{a}\left[1-\left(S / \delta_{a}\right)^{2}\right]
$$

where $\delta_{t}, \delta_{a}$ and $S$ are the true (corrected) line width (full width at half height, FWHH), the apparent (observed) line width and the spectral width, respectively. The scanning speed was $5 \mathrm{~cm}^{-1}$ per minute, and chart speed was 2 or $5 \mathrm{~cm}^{-1}$ per minute. A polarizer was placed in the path of the incident beam and also in the scattered beam. The parallel $I_{i}(\omega)$ and perpendicular $I_{\perp}(\omega)$ components were thus recorded independently. The spectra were recorded with slit widths ranging from 150 to $200 \mu$. Spectra of benzonitrile were recorded in solutions of $\mathrm{CCl}_{4}, \mathrm{CS}_{2}$, cyclohexane, cyclohexanol and tertiary butyl alcohol (TBA), having different viscosities.

All solutions used in this work were prepared using $1 \mathrm{ml}$ by volume of each solvent and ten percent by volume of each solute. The benzonitrile bands studied were the totally symmetric vibrations 2243 and $1000 \mathrm{~cm}^{-1}$, with a small depolarization factor. For the analysis, the high frequency side of each band of the solute in a given solvent was folded to give a symmetric peak. 


\section{Results and discussion}

(i) The normalized intensity distribution $I_{\text {iso }}(\omega)$ and $I_{\text {aniso }}(\omega)$ for the isotropic and anisotropic components of the benzonitrile $2243 \mathrm{~cm}^{-1}(\mathrm{C} \equiv \mathrm{N})$ stretch band are given in figures 1 and 2 and those for the $1000 \mathrm{~cm}^{-1}$ (ring breathing) band in figures 3 and 4 in the various solvents. The solvents have been chosen in such a way that the solvent bands do not interfere with solute bands.

(ii) In figures 5 and 6 , the rotational correlation function $C_{\text {rot }}(t)$ is plotted as a function of $t$ for the above two bands in different solvents. The solvent viscosities $\eta$ are indicated against each plot.

(ii) The half-widths $\Delta \omega_{1 / 2}$ of the bands for the isotropic and anisotropic components and the difference between the two which gives $\left(\Delta \omega_{1 / 2}\right)_{\text {rot }}$ as per $(7)$ are given for various solvents in table 1.

(iv) The $\tau_{\text {rot }}$ values calculated from $(\Delta \omega)_{1 / 2}$ and from the $1 / e$ th value of $C_{\text {rot }}(t)$ at $t=0$ are listed in table 2 , together with the rotational diffusion constant $\left(D_{\perp}\right)$ for all the solvents.

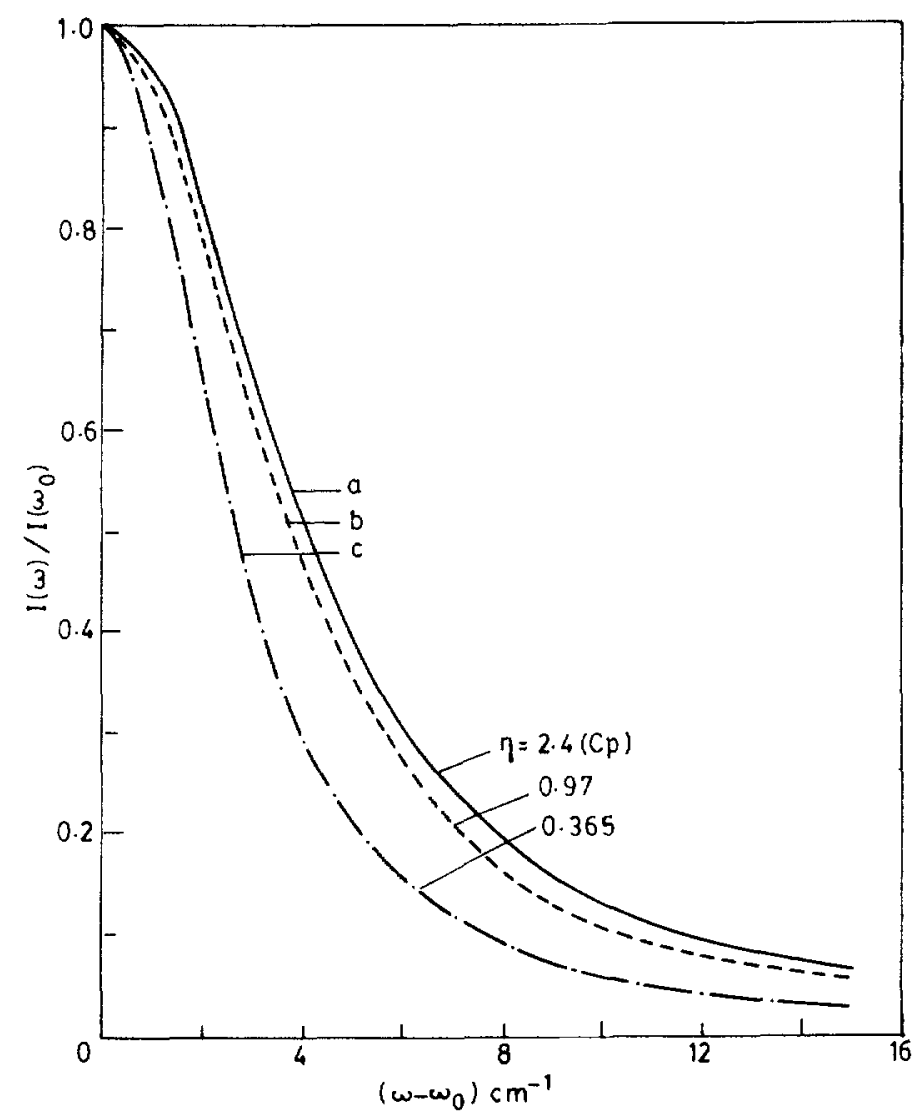

Figure 1. Normalized line shapes of $I_{\text {iso }}(\omega)$ for the $2243 \mathrm{~cm}^{-1}$ band of benzonitrile in (a) TBA, (b) $\mathrm{CCl}_{4}$ and (c) $\mathrm{CS}_{2}$. 


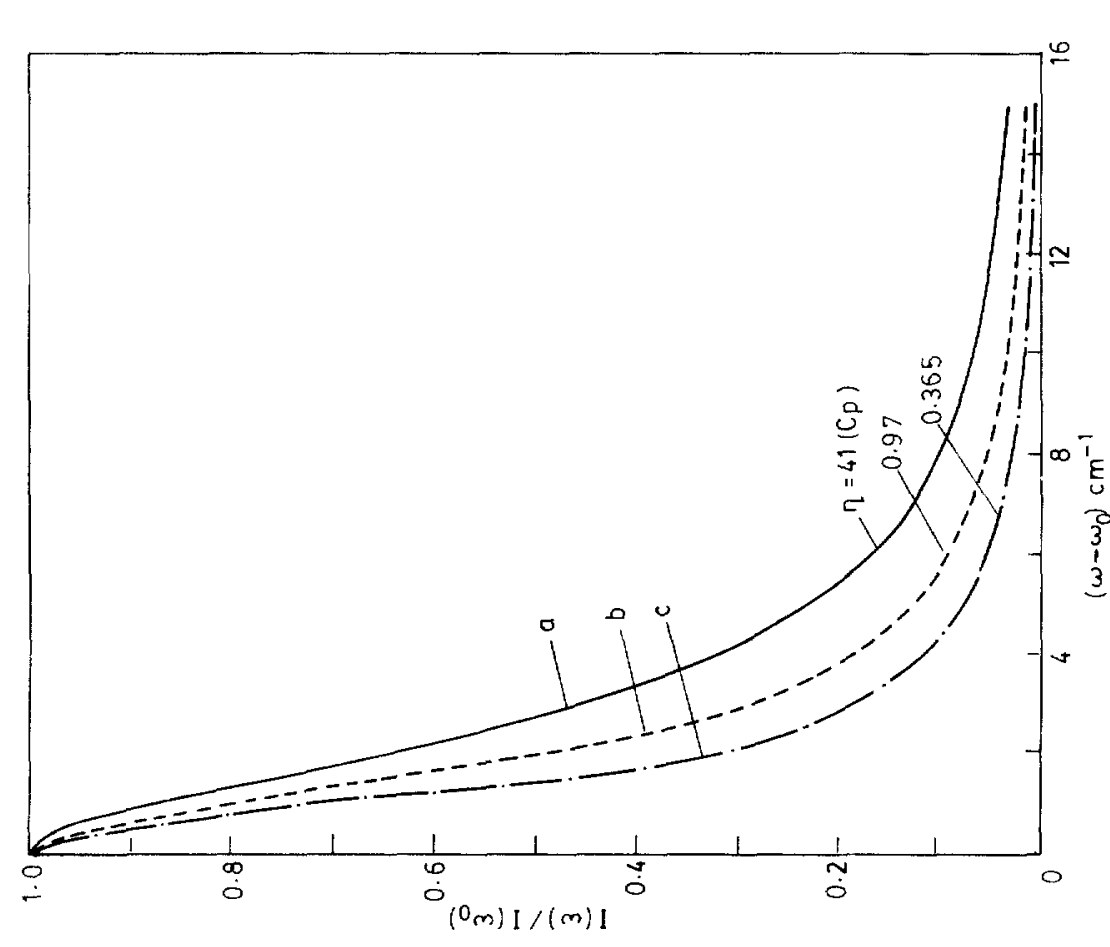

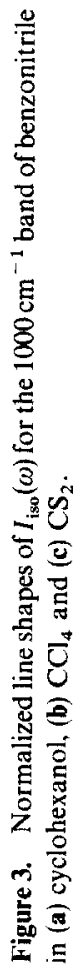

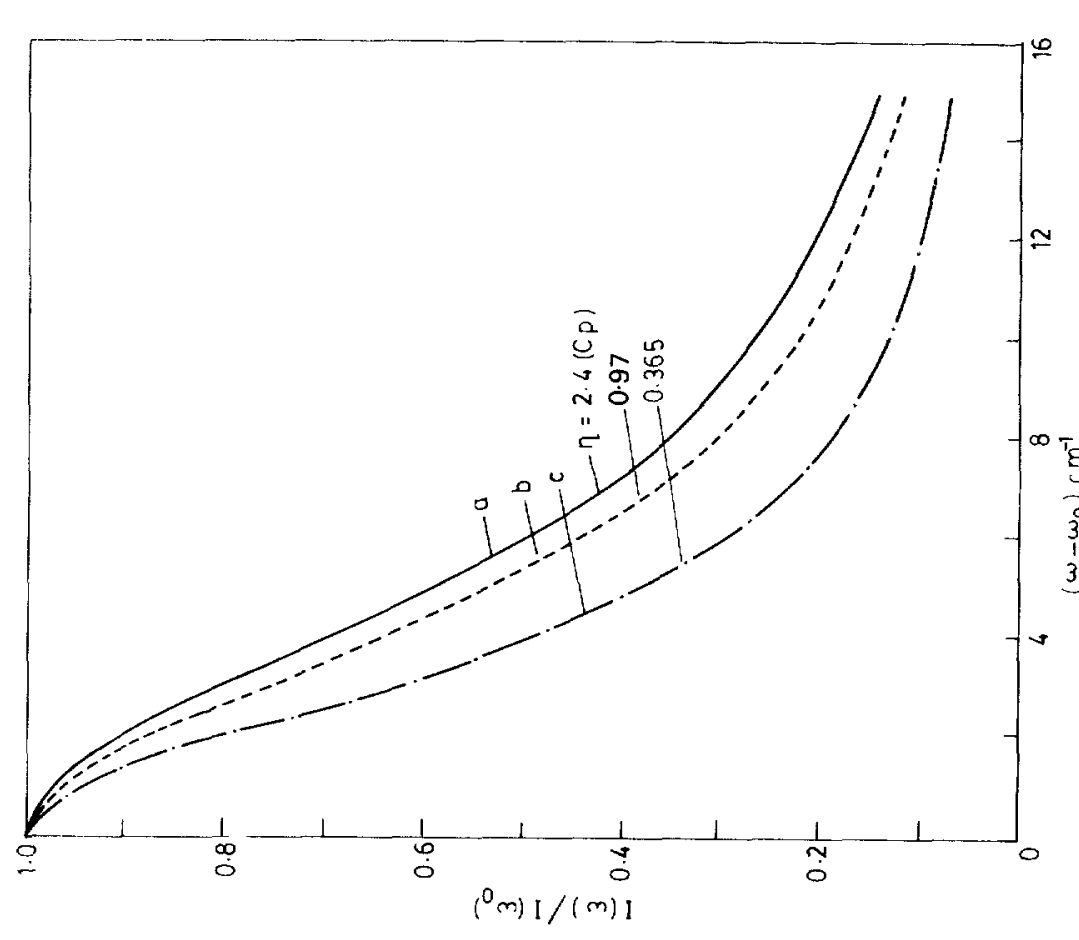

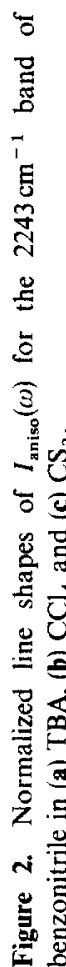



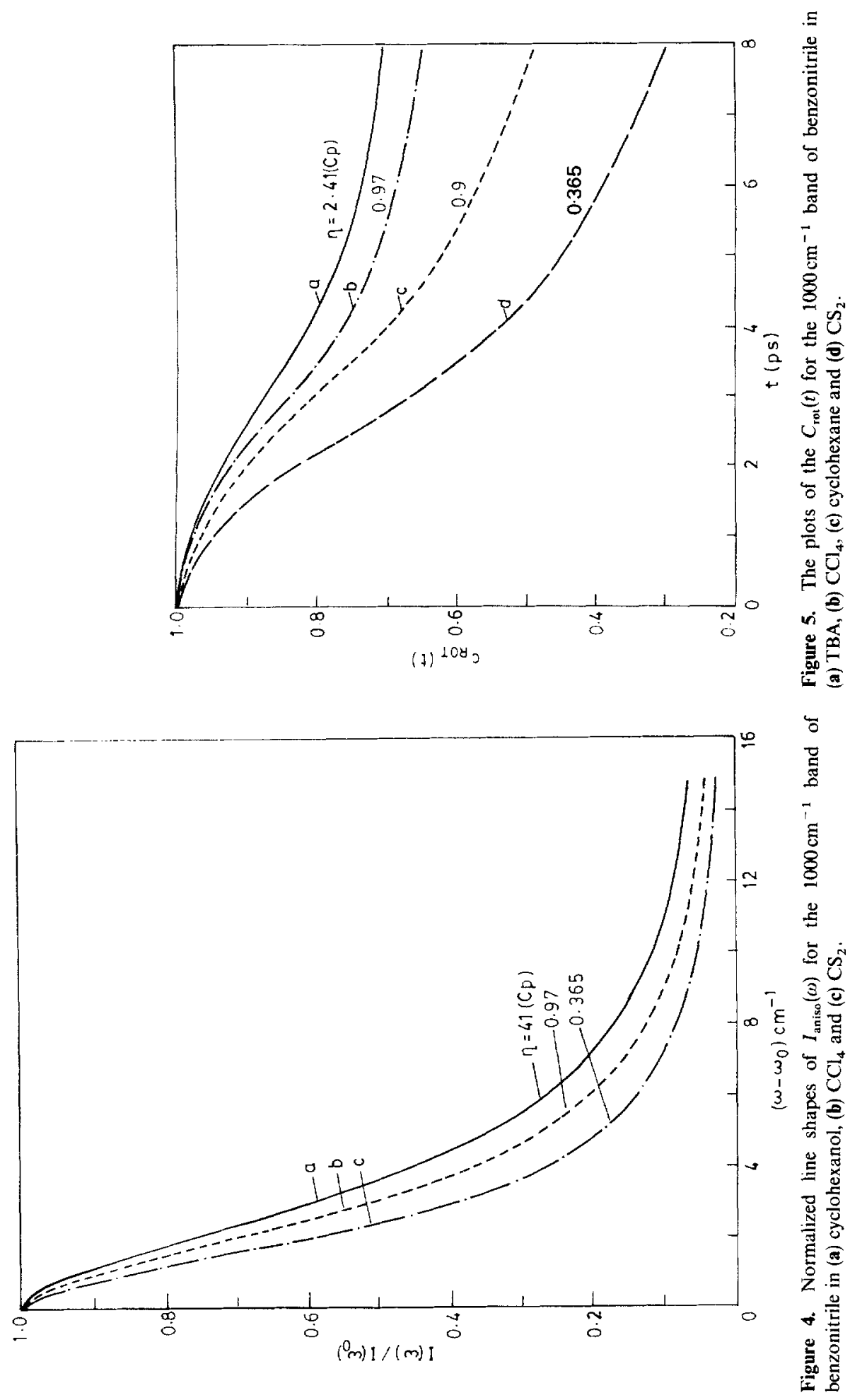


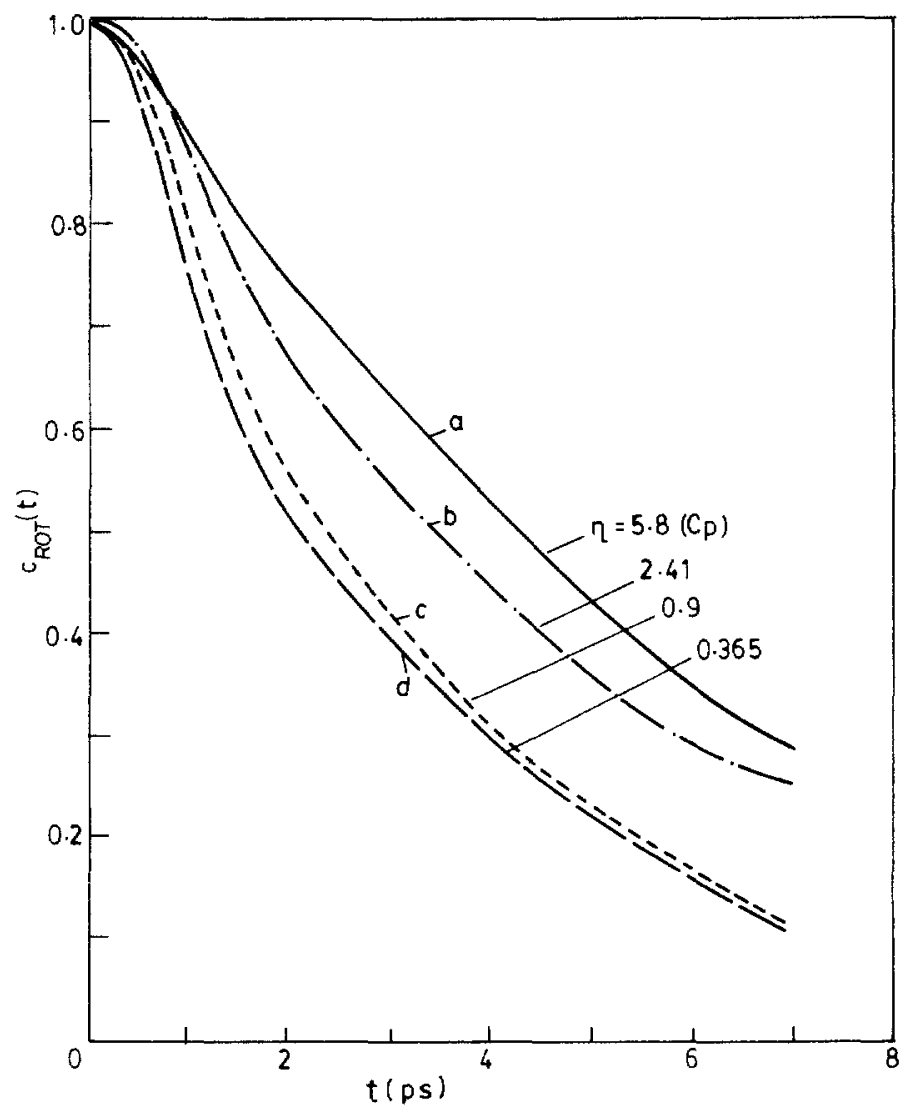

Figure 6. The plots of the $C_{\text {rot }}(t)$ for the $2243 \mathrm{~cm}^{-1}$ band of benzonitrile in (a) benzyl alcohol, (b) TBA, (c) cyclohexane and (d) $\mathrm{CS}_{2}$.

Table 1. Halfwidths of Raman bands of benzonitrile in different solvents. All values in $\mathrm{cm}^{-1}$.

\begin{tabular}{|c|c|c|c|c|c|c|}
\hline \multirow[b]{2}{*}{ Solvents } & \multicolumn{3}{|c|}{$2243 \mathrm{~cm}^{-1}$} & \multicolumn{3}{|c|}{$1000 \mathrm{~cm}^{-1}$} \\
\hline & $\Delta \omega_{\text {aniso }}$ & $\Delta \omega_{\text {iso }}$ & $\Delta \omega_{\text {ror }}$ & $\Delta \omega_{\text {aniso }}$ & $\Delta \omega_{\text {iso }}$ & $\Delta \omega_{\mathrm{rot}}$ \\
\hline $\mathrm{CS}_{2}$ & $7 \cdot 75$ & 6.00 & 1.75 & 1.88 & $1 \cdot 37$ & 0.51 \\
\hline $\begin{array}{l}\text { Cyclo- } \\
\text { hexane }\end{array}$ & $7 \cdot 75$ & $6 \cdot 25$ & $1 \cdot 50$ & 1.98 & $1 \cdot 53$ & 0.45 \\
\hline $\mathrm{CCl}_{4}$ & $8 \cdot 25$ & 7.00 & 1.25 & $2 \cdot 21$ & 1.83 & 0.38 \\
\hline TBA & 9.75 & 8.68 & 1.07 & 2.44 & $2 \cdot 10$ & $0 \cdot 34$ \\
\hline $\begin{array}{l}\text { Benzyl } \\
\text { alcohol }\end{array}$ & $10 \cdot 50$ & $9 \cdot 50$ & $1 \cdot 00$ & - & - & - \\
\hline $\begin{array}{l}\text { Cyclo- } \\
\text { hexanol }\end{array}$ & $10 \cdot 75$ & 1000 & 0.75 & $2 \cdot 88$ & $2 \cdot 58$ & 0.30 \\
\hline
\end{tabular}


Table 2. $\tau_{\text {rot }}$ in units of $10^{-12} \mathrm{~s}$ and $D_{\perp}$ in units of $10^{12} \cdot \mathrm{s}^{-1}$ for benzonitrile in different solvents.

\begin{tabular}{|c|c|c|c|c|c|c|}
\hline \multirow[b]{2}{*}{ Solvents } & \multicolumn{3}{|c|}{$2243 \mathrm{~cm}^{-1}$} & \multicolumn{3}{|c|}{$1000 \mathrm{~cm}^{-1}$} \\
\hline & $\begin{array}{c}\tau_{\mathrm{roe}} \\
\text { calculated } \\
\text { from FWHH }\end{array}$ & $\begin{array}{c}\text { Diffusion } \\
\text { constant } D_{\perp}\end{array}$ & $\begin{array}{c}\tau_{\text {rot }} \\
\text { calculated } \\
\text { from } 1 / e t h \\
\text { of } C_{\text {rot }}\end{array}$ & $\begin{array}{c}\tau_{\text {rot }} \\
\text { calculated } \\
\text { from FWHH }\end{array}$ & $\begin{array}{c}\text { Diffusion } \\
\text { constant } D_{\perp}\end{array}$ & $\begin{array}{c}\tau_{\text {rot }} \\
\text { calculated } \\
\text { from } 1 / e \text { th } \\
\quad \text { of } C_{\text {rot }}\end{array}$ \\
\hline $\mathrm{CS}_{2}$ & 3.03 & 0.0550 & 4.37 & $10 \cdot 40$ & 0.0160 & 10.85 \\
\hline $\begin{array}{l}\text { Cyclo- } \\
\text { hexane }\end{array}$ & 3.54 & 0.0471 & 4.77 & 11.79 & 0.0141 & 15.97 \\
\hline $\mathrm{CCl}_{4}$ & $4 \cdot 24$ & 0.0393 & $5 \cdot 76$ & 13.96 & 0.0119 & $17 \cdot 41$ \\
\hline TBA & 4.96 & 0.0336 & $7 \cdot 16$ & $15 \cdot 60$ & 0.0107 & $17 \cdot 98$ \\
\hline $\begin{array}{l}\text { Benzyl } \\
\text { alcohol }\end{array}$ & $5 \cdot 30$ & 0.0314 & $7 \cdot 37$ & - & -- & - \\
\hline $\begin{array}{l}\text { Cyclo- } \\
\text { hexanol }\end{array}$ & 7.07 & 0.0236 & $10 \cdot 21$ & 17.69 & $0-0094$ & $23 \cdot 70$ \\
\hline
\end{tabular}

The following observations and conclusions can be made from the results obtained.

(i) Figures 1 to 4 indicate that both the isotropic and anisotropic components of the Raman bands of benzonitrile are narrow for the $\mathrm{CS}_{2}$ solution and much larger for the TBA or cyclohexanol solutions, with the curves for $\mathrm{CCl}_{4}$ or cyclohexane lying in-between. This trend is in conformity with the viscosities of the solvents - the higher the viscosity, the more the widening of both the isotropic and anisotropic components. (ii) Figures 5 and 6 indicate that the $\mathrm{C} \equiv \mathrm{N}$ stretching mode at $2243 \mathrm{~cm}^{-1}$ and the ring breathing mode at $1000 \mathrm{~cm}^{-1}$, both belonging to the $a_{1}$ symmetry, have different correlation functions - the decay being much faster for the ring breathing mode. This happens for all the solvents. Detailed work on similar molecules is being carried out to understand why this happens.

(iii) $C_{\text {rot }}(t)$ falls faster as the viscosity decreases.

(iv) Table 1 shows that, in general, the isotropic line width increases as the viscosity of the solvent increases, whereas the anisotropic line width does not show significant variations. Tanabe and Hiraishi (1980b) have found similar results while studying solvent effects on chloroform. The larger variations for isotropic line widths are attributed to the increase in intermolecular interactions which affects the vibrational contribution to a greater extent than the rotational contribution.

(v) In table 2, the $\tau_{\text {rot }}$ values obtained from the $1 / \mathrm{eth}$ value of $C_{\text {rot }}(t)$ at $t=0$ are usually higher than those obtained from $\Delta \omega_{1 / 2}$. This shows that a strict exponential decay can not always be assumed.

(vi) The diffusion constants $D_{\perp}$ pertain to the rotational diffusion of the two-fold symmetry axis in benzonitrile as the bands considered, being of the $a_{1}$ species, arise from vibrations parallel to this axis.

\section{References}

Naffie L A and Peticolas W L 1972 J. Chem. Phys. 573145

Prakash R and Thyagarajan G 1982 J. Raman Spectrosc. 1273

Tanabe K and Hiraishi J 1980a Spectrochim. Acta A36 341

Tanabe K and Hiraishi J 1980b Adv. Mol. Relaxation Interaction Processes 16281 